\title{
Short-term fatty acid intervention elicits differential gene expression responses in adipose tissue from lean and overweight men
}

\author{
Marjan J. van Erk · Wilrike J. Pasman • \\ Heleen M. Wortelboer • Ben van Ommen • \\ Henk F. J. Hendriks
}

Received: 29 August 2008/Accepted: 13 November 2008/Published online: 26 November 2008

(C) The Author(s) 2008. This article is published with open access at Springerlink.com

\begin{abstract}
The goal of this study was to investigate the effect of a short-term nutritional intervention on gene expression in adipose tissue from lean and overweight subjects. Gene expression profiles were measured after consumption of an intervention spread (increased levels of polyunsaturated fatty acids, conjugated linoleic acid and medium chain triglycerides) and a control spread (40 g of fat daily) for 9 days. Adipose tissue gene expression profiles of lean and overweight subjects were distinctly different, mainly with respect to defense response and metabolism. The intervention resulted in lower expression of genes related to energy metabolism in lean subjects, whereas expression of inflammatory genes was down-regulated and expression of lipid metabolism genes was upregulated in the majority of overweight subjects. Individual responses in overweight subjects were variable and these correlated better to waist-hip ratio and fat percentage than BMI.
\end{abstract}

Keywords Subcutaneous adipose tissue $\cdot$ Microarray · Polyunsaturated fatty acids (PUFA) · Conjugated linoleic acid (CLA) · Medium chain triglycerides

Electronic supplementary material The online version of this article (doi:10.1007/s12263-008-0096-z) contains supplementary material, which is available to authorized users.

M. J. van Erk $(\bowtie)$ - W. J. Pasman · H. M. Wortelboer ·

B. van Ommen - H. F. J. Hendriks

Businessunit Biosciences, TNO Quality of Life, P.O. Box 360,

3700 AJ Zeist, The Netherlands

e-mail: marjan.vanerk@tno.nl

\section{Introduction}

Adipose tissue not only functions as a fat storage organ, it also has an important metabolic and endocrine function. Adipose tissue plays a role in insulin sensitivity and inflammatory status, e.g., through release of fatty acids and adipokines [1, 27]. In obesity, adipose tissue mass is increased and as a result, endocrine and metabolic functions of adipose tissue are affected. Moreover, obesity is associated with systemic inflammation due to macrophage infiltration in adipose tissue. Complications of obesity include insulin resistance, cardiovascular disease and metabolic syndrome $[1,38,39]$.

It has been shown that energy restriction, changes in dietary macronutrient composition and weight loss result in gene expression changes in adipose tissue from overweight or obese subjects $[6,22,35]$. The goal of this study was to investigate the effects of a short-term nutritional intervention on gene expression in adipose tissue and to investigate whether the gene expression response differs between lean and overweight subjects. Earlier, we showed that dietary effects can be monitored by gene expression profiling in white blood cells [32].

The nutritional intervention consisted of a spread with increased levels of medium chain triglycerides, short-chain polyunsaturated fatty acids (PUFA: C18:2 and C18:3) and conjugated linoleic acid (CLA: C18:2) (further referred to as intervention spread). This composition was chosen to elicit an effect on food intake and satiety. Medium chain triglycerides, PUFA and CLA are reported to have satiating power $[13,18,29]$. The two spreads did not differ in their effect on satiety (described in detail elsewhere: Pasman et al., in preparation). To a large extent, it is unknown how the fatty acids can influence food intake, energy expenditure and eventually body mass [17]. Analysis of adipose 
tissue gene expression is of interest because of its central role in fat and energy metabolism in response to nutritional interventions and because of its role in inflammation. Furthermore, PUFA have been reported to influence adipose tissue function $[5,21]$ and CLA can play a role in reduction of adipose tissue mass [3, 37, 40].

We found that in adipose tissue fatty acid transporter gene expression was slightly higher in lean subjects and slightly lower in overweight subjects in response to the intervention spread compared to the control spread. Also, fatty acid uptake showed a tendency to change in response to the intervention (details in Pasman et al., in preparation). Here we study in greater detail the response of adipose tissue to the different fatty acid intake by whole genome gene expression profiling of subcutaneous adipose tissue biopsies taken from the subjects after consumption of the intervention or control spread with an equal amount of fat for 9 days.

Effects of the short-term nutritional intervention were studied both in overweight and lean subjects, by focusing on enriched functional groups of genes and putting these in the context of the intervention, thereby aiming to shed a light on the potential mechanisms of action of the fatty acids.

\section{Subjects and methods}

Study design and subjects

The study was conducted at TNO Quality of Life, where subjects were recruited from a pool of volunteers. Each subject was informed about the study orally and in writing before they signed the informed consent forms. Subjects met the inclusion criteria: (1) healthy male subjects (lean: BMI between 18 and $25 \mathrm{~kg} / \mathrm{m}^{2}$ or overweight/obese: BMI between 27.5 and $35 \mathrm{~kg} / \mathrm{m}^{2}$ ), (2) normal Dutch eating pattern and used to consuming breakfast, (3) non-restrained eaters according to the Dutch Eating Behavior Questionnaire (lean: $<2.5$ and overweight/obese: $<3.25$ ) [33, 34]. Subjects with a disorder, using medication or having a history of medical or surgical events that may have affected study outcomes were excluded.

A total of 25 subjects (all of Dutch ancestry) were eligible of which 20 subjects were randomly selected as well as four reserves. Ten lean (BMI mean \pm SD $21.2 \pm$ $1.5 \mathrm{~kg} / \mathrm{m}^{2}$, age range $19-41$ years) and ten overweight men (BMI $29.7 \pm 1.9 \mathrm{~kg} / \mathrm{m}^{2}$, age range $21-58$ years) started and completed the study. Body fat percentage was $12.2 \pm 4.2 \%$ in the lean sub-group compared to $27.8 \pm$ $3.7 \%$ in the overweight sub-group. Waist circumference was $79 \pm 6 \mathrm{~cm}$ in the lean sub-group compared to $102 \pm 8 \mathrm{~cm}$ in the overweight sub-group and waist-hip ratio (WHR) was $0.86 \pm 0.05$ in the lean sub-group compared to $0.96 \pm 0.07$ in the overweight subgroup.

The study was approved by the Medical Ethics Committee of the University Medical Centre of Utrecht (20 September 2005) and conducted according to the ICH Guideline for Good Clinical Practice in October-November 2005 .

The study was designed as a randomized cross-over, placebo-controlled, double blind study. Each subject received two treatments. The treatment period lasted nine days and the wash-out period was 2 weeks. The two treatments consisted of a spread containing fatty acids known for their satiating effect [increased levels of medium chain triglycerides, short-chain polyunsaturated fatty acids (PUFA: C18:2 and C18:3) and conjugated linoleic acid (CLA: C18:2) (further referred to as intervention spread)] and a control spread containing fatty acids normally eaten with breakfast. The exact composition of the spreads is presented in Table 1 . Blends with the fatty acids of interest were mixed by Lipid Nutrition (Wormerveer, The Netherlands). Romi Smil (Heerenveen, The Netherlands) prepared the spreads. The subjects used the spread with breakfast and lunch; 26 grams in the morning, and $26 \mathrm{~g}$ in the afternoon (in total $52 \mathrm{~g}$ of spread daily, containing $40 \mathrm{~g}$ of fat). The spreads replaced the spread normally consumed in the diet. The amount of fat supplied in the spread was similar in both interventions and energy intake was kept constant, resulting in a difference in fatty acid composition intake only.

No carry-over effect of treatments was found for bodyweight (data not shown).

\section{Adipose tissue biopsies}

Two fat biopsies were taken from each subject, one after consumption of the placebo spread for 9 days and one after consumption of the intervention spread for 9 days. Biopsies were taken in the evening and time of sampling was

Table 1 The composition of the spreads

\begin{tabular}{lll}
\hline & $\begin{array}{l}\text { Intervention } \\
\text { spread (\%) }\end{array}$ & $\begin{array}{l}\text { Control } \\
\text { spread (\%) }\end{array}$ \\
\hline Lauric acid (MCT, C12:0) (and C14:0) & 17.2 & 0 \\
Palmitic acid (C16:0) & 17.9 & 17.5 \\
Stearic acid (C18:0) & 3 & 10 \\
Oleic acid (C18:1) & 4.5 & 40 \\
Conjugated linoleic acid (C18:2) & 12.4 & 0 \\
Linoleic acid (C18:2) & 40.3 & 30 \\
Pinolenic acid (C18:3) & 2.5 & 0 \\
Other fatty acids & 2.2 & 2.5 \\
\hline
\end{tabular}


kept constant as much as possible for the two treatments. Subcutaneous adipose tissue was obtained from the abdominal region by needle aspiration under local anesthesia using $2 \times 50 \mathrm{ml} 8.4 \% \mathrm{NaHCO}_{3}, 0.05 \%$ lidocaine and $1.0 \mathrm{mg} / \mathrm{ml}$ epinephrine (according to Kolaczynski et al. [16]). Part of the fat suspension was immediately frozen in liquid nitrogen and stored at $-80^{\circ} \mathrm{C}$ for RNA isolation.

RNA isolation, labeling and hybridization

RNA was isolated by homogenizing the adipose tissue biopsies in Trizol (Life Technologies S.A., Merelbeke, Belgium). After chloroform extraction the RNA isolation was continued using NucleoSpin RNA II kit (Bioké, Leiden). The protocol included a DNase digestion step.

The isolated RNA samples were processed according to Affymetrix protocols by Scienion Ag (Berlin, Germany). In brief, RNA quality and integrity were verified using the RNA 6000 Nano assay on the Agilent 2100 Bioanalyzer (Agilent Technologies), resulting in RNA samples from ten lean and eight overweight subjects to be included in the microarray analysis (RNA samples from two overweight subjects did not pass the RNA quality control).

Next, $1.5 \mu \mathrm{g}$ of high-quality total RNA was used with the Affymetrix Eukaryotic One-Cycle Target Labeling and Control reagents to generate Biotin-labeled antisense cRNA. The labeled cRNA was further used for the hybridization to the NuGO Affymetrix Human Genechip NuGO_Hs1a520180 (custom designed by the European Nutrigenomics Organization NuGO, consisting of 23,941 probesets including 71 control probesets, for details see http://blog.bigcat.unimaas.nl/ martijn/NuGO/). After an automated process of washing and staining, absolute values of expression were calculated from the scanned array using the Affymetrix GCOS software.

\section{Data analysis}

Quality control of microarray data was performed using BioConductor packages (a.o. simpleaffy and affyplm, through the NuGO MadMax pipeline: https://madmax. bioinformatics.nl). One microarray did not pass quality control criteria due to high background values and low percentage of present calls. Therefore, microarray data for nine lean and eight overweight subjects were included in data analysis.

Raw signal intensities (from CEL files) were normalized using the GCRMA algorithm (gc-rma slow). Probesets were filtered on at least one present call and at least one expression value above 5 (resulting in a set of 15,762 probesets). Expression data were log-transformed (base 2). The cross-over design of the study ensured measurement of gene expression profiles in adipose tissue of each subject after consumption of the placebo spread and after consumption of the intervention spread, allowing for analysis of gene expression changes in response to intervention within each subject [calculated as $2 \log$ ratio (intervention vs placebo)].

First, effects of BMI (lean sub-group or overweight subgroup), treatment (intervention spread or control spread) as well as the interaction component BMI $\times$ Intervention was tested using two-way ANOVA in SAS (SAS Institute Inc., Cary, USA). The null hypotheses (no difference) were rejected at the level of 0.01 of probability. When testing 15,762 probesets with these conditions, 158 probesets are expected to be called significant.

Second, a paired $t$ test for treatment effect in the lean and in the overweight subgroup was performed in BRB ArrayTools (software for microarray data analysis developed by Dr. Richard Simon and Amy Peng Lam, http://linus.nci.nih.gov/BRB-ArrayTools.html), using multiple testing correction by estimation of false discovery rate (FDR). Threshold for significance was set by taking into account $P$ value $(<0.01)$ as well as estimated FDR $(<20 \%)$. Finally, in case of responders and non-responders within a sub-group, differentially expressed genes were selected based on showing a consistent response in the majority of the subjects (defined as $\geq 25 \%$ expression change up in more than $50 \%$ of the subjects or $\geq 25 \%$ expression change down in more than $50 \%$ of the subjects).

Subsets of differentially expressed genes were analyzed further in GenMAPP to find functional groups of genes (based on Gene Ontology) that were overrepresented in the subsets [7]. Other tools used for data analysis include Gene Set Enrichment Analysis [31] and T-profiler [2] for enrichment analysis of functional groups of genes. The tissue-specific gene sets were derived from SymAtlas (http://symatlas.gnf.org/SymAtlas/) [30] and from Lyons et al. [20]. Cluster and treeview (http://rana.lbl.gov/ EisenSoftware.htm, [8]) were used for generation of cluster trees, using average linkage clustering and uncentered correlation as similarity metric. A biological network was generated in MetaCore version 4.3 (GeneGo Inc., St Joseph, MI, USA). MetaCore ${ }^{\mathrm{TM}}$ is based on a proprietary manually curated database of human protein-protein, protein-DNA and protein compound interactions, metabolic and signaling pathways and the effects of bioactive molecules in gene expression. Only curated interactions were used.

Data from the placebo intervention were used for comparison of gene expression levels in adipose tissue from lean and overweight subjects.

The gene expression data are available through ArrayExpress, a public repository for microarray data (http:// www.ebi.ac.uk/arrayexpress/, accession numer E-TABM377). 


\section{Results}

Subcutaneous adipose tissue gene expression profiles of lean and overweight subjects were distinctly different (3,069 probesets selected with $P<0.01$ for BMI-effect; Fig. 1). An overview of enriched functional groups of genes (based on Gene Ontology) in this set of 3,069 probesets is shown in Table 2. Genes involved in energy metabolism were expressed at a higher level in adipose tissue from lean subjects (functional groups 'generation of precursor metabolites and energy', 'lipid metabolism', 'glucose metabolism'). Furthermore, genes encoding mitochondrial proteins were expressed at a higher level in adipose tissue from lean subjects than in adipose tissue from overweight subjects.

On the other hand, genes involved in defense response, intracellular signaling and cell adhesion were expressed at higher levels in adipose tissue from overweight subjects than in adipose tissue from lean subjects. Within these groups of genes, gene expression of specific macrophage markers [e.g., CD14, CD163 and macrophage scavenger receptor 1 (MSR1)] was significantly higher in adipose tissue of overweight subjects $(P<0.001$ for BMI-effect in two-way ANOVA), pointing towards presence of macrophages or inflammatory cells in the adipose tissue biopsies from overweight subjects. This is further illustrated in supplementary Fig. 1, which clearly shows higher expression of genes specific for CD14-positive monocytes in adipose tissue from seven overweight subjects than in

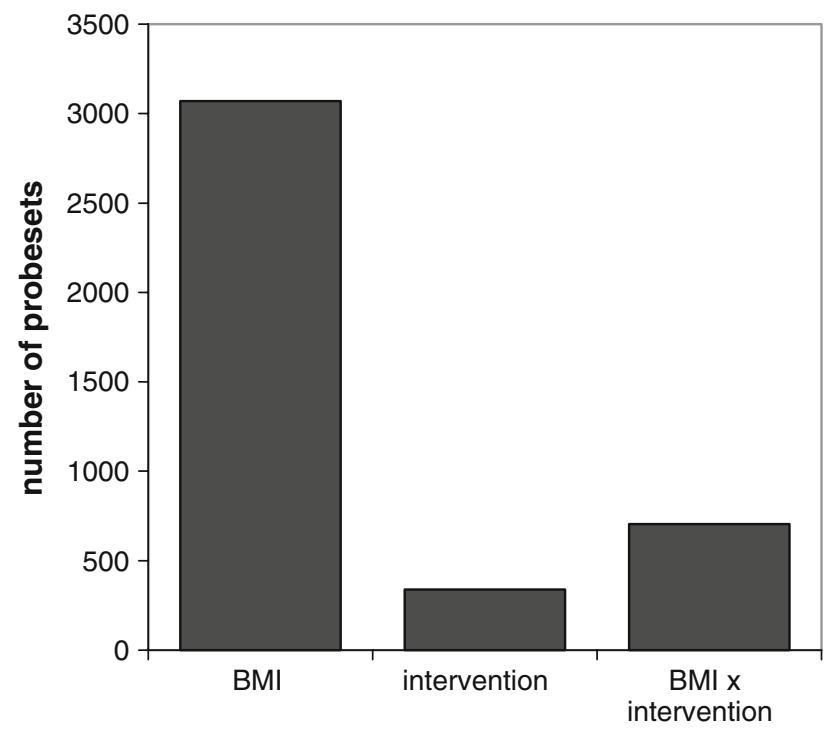

Fig. 1 Results of 2-way ANOVA investigating effect of BMI [lean $(n=9)$ or overweight $(n=8)$ group], intervention (intervention spread or placebo) and BMI $\times$ intervention interaction. Threshold for significance was set at $P<0.01$. Testing 15,762 probesets with $P$ value threshold of 0.01 would result in 158 probesets detected as significant by chance adipose tissue from lean subjects. Enrichment analysis of tissue-specific gene sets, by comparing the lean sub-group to overweight sub-group (GSEA) and by analyzing overweight subjects individually (T-profiler), showed that this set of genes specific for CD14-positive monocytes was significantly enriched in the adipose tissue from overweight subjects compared to lean subjects and that this gene set was among the two most enriched gene sets in seven out of the eight overweight subjects. Other inflammatory markers that were expressed at higher level in adipose tissue from overweight subjects included leptin, complement factor D (CFD), interleukin receptor 6 (IL6R), interleukin receptor 10 alpha (IL10RA), interleukin 1 receptor antagonist (IL1RN) and a number of interleukins (IL15, IL17D, IL18) (data not shown).

Consumption of the intervention spread resulted in a differential gene expression response in adipose tissue from lean and overweight subjects, as indicated by the BMIintervention interaction effect (706 probesets selected with $P<0.01$ for BMI $\times$ intervention interaction effect; Fig. 1). When plotting the expression changes (intervention spread versus control spread) of this set of 706 genes in the lean and overweight subjects (Fig. 2), it can be seen that the expression changes are more pronounced (larger fold changes) in the lean subjects than in the overweight subjects. In addition, statistical analysis of treatment effects in the lean and overweight sub-group individually resulted in no probesets significantly differentially expressed due to the intervention in overweight subjects compared to 813 differentially expressed probesets due to the intervention in lean subjects.

Enrichment analysis of the set of differentially expressed genes in lean subjects indicated that in lean subjects consumption of the intervention spread led to down-regulation of genes involved in functional groups indicated as 'mitochondrion', 'generation of precursor metabolites and energy', 'lipid metabolism', 'carbohydrate metabolism' (Table 3), together pointing towards a general effect on energy metabolism. These selected 'energy metabolism' genes showed considerable variation in their response among the overweight subjects (supplementary Fig. 2).

The results indicate that in the overweight subjects the response to the intervention is less strong and more variable than in the lean subjects. We further explored the possibility that a selection of the overweight subjects did show a consistent gene expression response for a number of genes or processes. Therefore, genes were selected based on showing a consistent response in the majority of the subjects (defined as $\geq 25 \%$ expression change up in more than $50 \%$ of the subjects or $\geq 25 \%$ expression change down in more than $50 \%$ of the subjects). In total, 501 probesets fulfilled these criteria. Enriched functional groups in this set of genes were 'lipid metabolism', 'response to stress', 
Table 2 Enriched functional groups (based on Gene Ontology) in total set of differentially expressed genes in lean versus overweight subjects (3,069 probesets), in genes expressed at high levels in lean than in overweight (1,325 probesets) and in genes expressed at higher levels in lean than overweight (1,744 probesets)

\begin{tabular}{|c|c|c|c|c|c|c|c|c|}
\hline \multirow{2}{*}{$\begin{array}{l}\text { GO } \\
\text { group }\end{array}$} & \multirow[t]{2}{*}{ Functional group of genes } & \multirow{2}{*}{$\begin{array}{l}\text { Number of genes } \\
\text { measured }\end{array}$} & \multicolumn{2}{|c|}{ All genes } & \multicolumn{2}{|c|}{ Higher in lean } & \multicolumn{2}{|c|}{ Higher in overweight } \\
\hline & & & $\begin{array}{l}\text { Percent } \\
\text { changed }\end{array}$ & $\begin{array}{l}Z \\
\text { score }^{\mathrm{a}}\end{array}$ & $\begin{array}{l}\text { Percent } \\
\text { changed }\end{array}$ & $\begin{array}{l}Z \\
\text { score }\end{array}$ & $\begin{array}{l}\text { Percent } \\
\text { changed }\end{array}$ & $\begin{array}{l}Z \\
\text { score }\end{array}$ \\
\hline $\mathrm{CC}$ & Mitochondrion & 522 & 38.5 & 14.4 & 34.3 & 25.7 & & \\
\hline $\mathrm{BP}$ & Generation of precursor metabolites and energy & 508 & 29.5 & 8.6 & 21.5 & 13.6 & & \\
\hline $\mathrm{BP}$ & Oxidative phosphorylation & 77 & 36.4 & 4.9 & 31.2 & 8.6 & & \\
\hline $\mathrm{BP}$ & Lipid metabolism & 503 & 27.6 & 7.3 & 17.9 & 10.2 & & \\
\hline $\mathrm{BP}$ & Carbohydrate metabolism & 392 & 25.5 & 5.3 & 12.5 & 4.7 & 13.0 & 2.6 \\
\hline $\mathrm{BP}$ & Glucose metabolism & 77 & 28.6 & 3.0 & 22.1 & 5.4 & & \\
\hline $\mathrm{BP}$ & Mitochondrial transport & 17 & 41.2 & 2.9 & 41.2 & 5.7 & & \\
\hline $\mathrm{BP}$ & Cell adhesion & 502 & 19.1 & 2.0 & & & 13.3 & 3.3 \\
\hline $\mathrm{BP}$ & Cell-matrix adhesion & 60 & 28.3 & 2.6 & & & 25.0 & 4.2 \\
\hline $\mathrm{BP}$ & Defense response & 723 & 24.2 & 6.3 & & & 21.5 & 11.8 \\
\hline $\mathrm{BP}$ & Inflammatory response & 183 & 26.2 & 3.8 & & & 24.6 & 7.2 \\
\hline $\mathrm{BP}$ & Response to stress & 829 & 20.6 & 3.8 & & & 17.0 & 8.0 \\
\hline $\mathrm{BP}$ & Intracellular signaling cascade & 930 & 19.1 & 2.8 & & & 13.8 & 5.0 \\
\hline $\mathrm{BP}$ & I-kappaB kinase/NF-kappaB cascade & 98 & 28.6 & 3.4 & & & 22.4 & 4.5 \\
\hline MF & Scavenger receptor activity & 28 & 32.1 & 2.4 & & & 32.1 & 4.2 \\
\hline
\end{tabular}

Enrichment analysis was performed in GenMAPP, by comparing the three selected sets of genes (all genes differentially expressed in lean versus overweight subjects, genes expressed at higher levels in lean than in overweight subjects and genes expressed at higher levels in overweight than in lean) to all genes on the microarray. Data from the placebo intervention were used for comparison of gene expression levels in adipose tissue from lean and overweight subjects

$G O$ Gene Ontology groups, $C C$ cellular component, $B P$ biological process, $M F$ molecular function

a All $Z$ scores in table: $P<0.05$ (PermuteP)

Fig. 2 Overview of expression changes (intervention spread vs. control spread) of 706 probesets $(P<0.01$ for $\mathrm{BMI} \times$ treatment effect) in nine lean $(L$ left side $)$ and eight overweight $(O$ right side) subjects

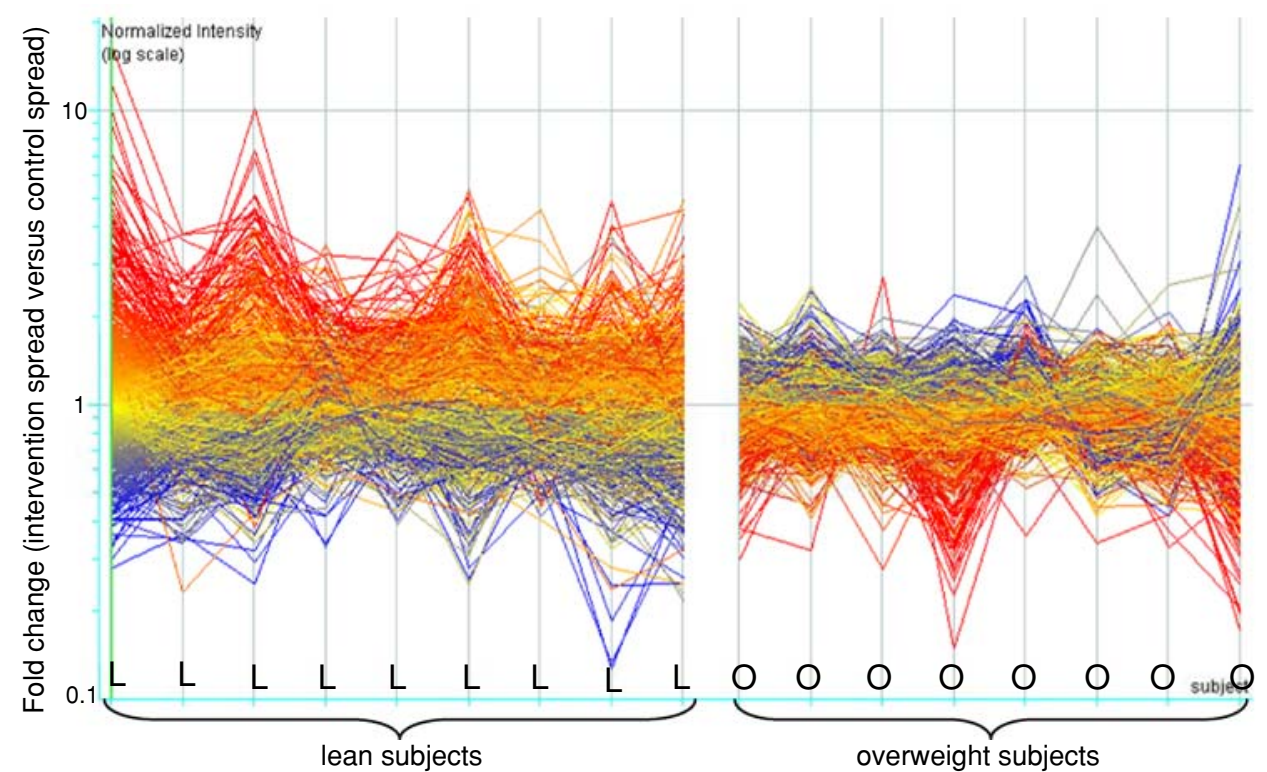

'extracellular matrix', 'apoptosis' and 'cell adhesion'. Clustering of the genes in these functional groups (Fig. 3) illustrate variation in response among the overweight subjects, but also show that six of the overweight subjects show a similar response for most functional groups, whereas two other respond differently. Interestingly, expression of a number of the lipid metabolism and inflammatory genes (response to stress) in the clusters was 
Table 3 Enriched functional groups (based on Gene Ontology) in set of differentially expressed genes (813 probesets) and in sub-set of downregulated genes ( 509 probesets) in sub-group of lean subjects in response to treatment

\begin{tabular}{|c|c|c|c|c|c|c|}
\hline \multirow[t]{2}{*}{ GO group } & \multirow[t]{2}{*}{ Functional group of genes } & \multirow{2}{*}{$\begin{array}{l}\text { Number of } \\
\text { genes measured }\end{array}$} & \multicolumn{2}{|l|}{ All } & \multicolumn{2}{|l|}{ Down-regulated } \\
\hline & & & Percent changed & $Z$ score & Percent changed & $Z$ score \\
\hline $\mathrm{CC}$ & Mitochondrion & 522 & 6.5 & 3.1 & 5.7 & 4.1 \\
\hline $\mathrm{BP}$ & Generation of precursor metabolites and energy & 508 & 6.9 & 3.5 & 5.5 & 3.7 \\
\hline $\mathrm{BP}$ & Lipid metabolism & 503 & 6.2 & 2.7 & 5.8 & 4.0 \\
\hline $\mathrm{BP}$ & Carbohydrate metabolism & 391 & 6.1 & 2.3 & 5.9 & 3.7 \\
\hline $\mathrm{BP}$ & Glucose metabolism & 77 & 10.4 & 2.9 & 10.4 & 4.0 \\
\hline $\mathrm{BP}$ & Mitochondrial transport & 17 & 23.5 & 4.2 & 23.5 & 5.1 \\
\hline $\mathrm{BP}$ & Regulation of cell cycle & 367 & 6.27 & 2.36 & 4.9 & 2.4 \\
\hline $\mathrm{BP}$ & Complement activationl, classical pathway & 22 & & & 13.6 & 3.0 \\
\hline $\mathrm{BP}$ & Oxygen and reactive oxygen species metabolism & 65 & & & 7.7 & 2.4 \\
\hline
\end{tabular}

Enrichment analysis was performed in GenMAPP, by comparing the two selected sets of genes (all genes differentially expressed in lean subjects in response to intervention and sub-set of down-regulated genes in lean subjects in response to treatment) to all genes on the microarray

$G O$ Gene Ontology groups, $C C$ cellular component, $B P$ biological process

a All $Z$ scores in table: $P<0.05$ (PermuteP)

significantly different in overweight compared to lean subjects (BMI-effect) and changed in response to consumption of the intervention spread in direction of expression levels in lean subjects (genes marked with asterisk in Fig. 3).

Finally, individual response in overweight subjects was studied on level of functional groups of genes, using T-profiler [2], resulting in an enrichment score for functional groups in individual subjects based on gene expression changes in response to the intervention. A number of functional gene groups showed considerable variation in response among the overweight subjects, with up-regulation in one or more subjects and down-regulation in one or more other subjects. These functional gene groups include 'mitochondrion', 'cell adhesion' and 'extracellular matrix', 'immune response' and 'inflammatory response'. Interestingly, the individual response with regard to these functional groups correlated significantly to obesity phenotype markers WHR or fat percentage (Fig. 4). WHR correlated negatively with response of mitochondrial genes and positively with response of genes involved in cell adhesion. In addition, fat percentage correlated positively with response of inflammatory genes.

\section{Discussion}

To our knowledge, this study is one of the first to perform large-scale gene expression analysis in human adipose tissue biopsies in response to a short-term nutritional intervention, in both lean and overweight subjects. The study is exploratory in nature and describes the results on a pathway or functional group level with interpretation in relation to the intervention and literature data.

Our study data indicated that a considerable part of the differences in gene expression profiles could be attributed to the difference in BMI between the two sub-groups. Expression of inflammation-related genes was significantly higher in subcutaneous adipose tissue biopsies from overweight subjects than those from lean subjects. Specifically, markers specific for monocytes in blood were significantly higher expressed in the adipose tissue from overweight subjects, as was shown by different enrichment analysis approaches. As tissue macrophages originate from monocytes in blood that migrate into tissue, this finding is consistent with increased macrophage content of adipose tissue from overweight subjects, as previously described by others [38]. In addition to macrophage markers, also expression of specific adipokines was different between lean and overweight subjects. Consistent with other reports $[14,23]$, expression of leptin was higher and expression of adiponectin was lower (although not significantly) in adipose tissue from overweight subjects than in adipose tissue from lean subjects.

Expression of metabolism-related genes and genes encoding mitochondrial proteins was higher in subcutaneous adipose tissue from lean subjects than in adipose tissue from overweight subjects. A recent study of transcription profiles in adipose tissue from monozygotic twins discordant for BMI also reported lower expression of genes related to energy metabolism in obese subjects [26]. In addition, they report a reduction in mitochondrial DNA in adipose tissue from obese subjects. Overall, this indicates a 
Fig. 3 Cluster maps of genes in biological processes enriched in set of genes with consistent expression change $(\geq 25 \%)$ in the majority of overweight subjects. Expression changes (2log ratio) of intervention spread versus placebo spread in eight overweight subjects were submitted to gene and sample clustering (using average linkage clustering and uncentered correlation as similarity metric). Red color indicates up-regulation of expression in response to intervention spread compared to placebo spread. Green color indicates down-regulation of expression in response to intervention spread compared to placebo spread. Asterisks are significantly different expression in overweight compared to lean subjects (BMI-effect) and expression changed in response to the intervention spread in direction of expression levels in lean subjects (colour in online)

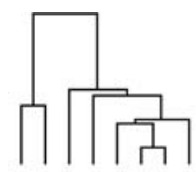

Response to stress

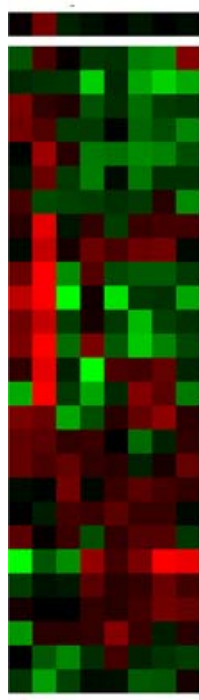
POLD 3 (212836 at) PLAT (201860 $\mathrm{s}$ at) CYGB (226632 at) CCL14 (205392 s at) RPS6KA5 (204635 at) CRY2 (NuGO eht0263762 at) Vha (202112 at) CD83 (204440 at) CCL 8 (NuGO eht 0225840 at) ITGB2 (202803 s at) *IIIRW (212657 $\mathrm{s}$ at) * STAB1 (38487 at) * MaRCO (NuGO eht032709? at) * CTGF (209101 at) CXCL2 (209774 $x$ at) * LBP (HuGo eht021740? at) BPP NuGo eht 021740? ARF 1 (210125 $\mathrm{s}$ at) O2TVT4 HUMAN (1554741 s at) FGF 10 (NuGO eht0264664 at) WP 872431.2 (WuGo eht $0317402 \mathrm{~s}$ at FOS (209189 at) STIP1 (213330 s at) SFPO (201585 $s$ at) RAD21 (20060? s at) PP 21 (2007s at CIDER (NuGO eht 0342845 at)

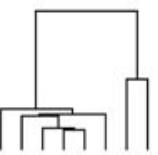

\section{Lipid metabolism}
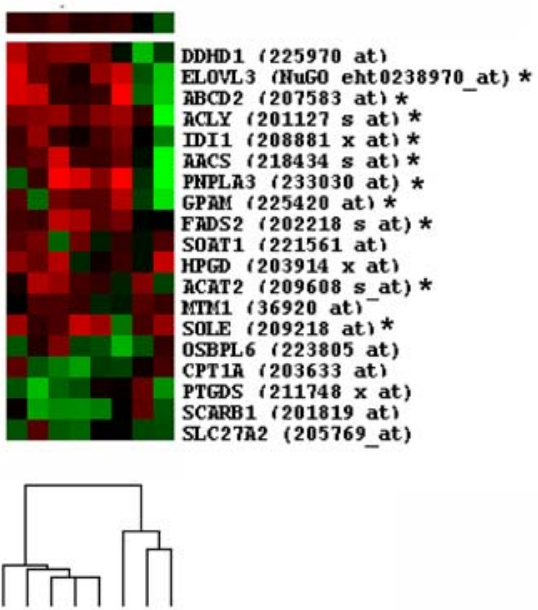

Extracellular matrix \& cell adhesion

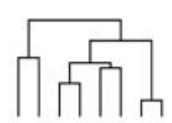

apoptosis

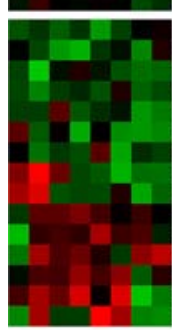
TIm1 1201449 at PAWR (204004 at) CIDE (NuGO eht 0342845 at) STK4 (243981 at) APAF1 (204859 $\mathrm{s}$ at) SCARB1 (201819 at) EP300 (213579 s at) Trab (202803 s at) TPI 5 (201686 at) API5 $201686 \mathrm{x}$ at) RZD21 (200607 s at) YWhEA (201020 at) ITGB3BP (205176 s_at FDRK3 210148 at)

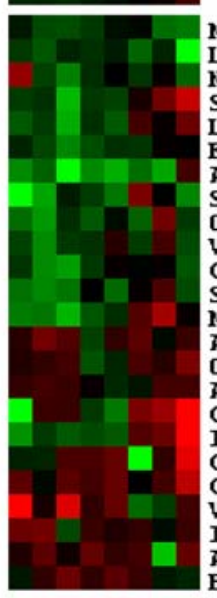

MPP27 (HuGo eht0260229 at) LTBP1 $(202729 \mathrm{~s}$ at) ISP 066013.1 (227870 at) STAB1 (38487 at) LAMB 2 (216264 $\mathrm{s}$ at) FFBPP4 (212232 at) FDAMTS18 (230040 at) SMOC2 (223235 s at) 016473 HURA (208609 s at) Vhe (202112 at) CPXI2 (226824 at) SCARB1 (201819 at) MFGE 8 (210605 $\mathrm{s}$ at) ADARTS1 (222162 $\mathrm{s}$ at) OMD (HuGo eht 0247535 at) ADPM12 (NuG0 eht 0278082 at CHI 3L1 (209395 at) ITGB2 (202803 $\mathrm{s}$ at) CTGF (209101 at) CDH11 (207173 $x$ at) VCAM1 (203868 $\mathrm{s}$ at) ITGB3BP (205176 $\mathrm{s}$ at) ARHGAP5 (1552627 a at) BYSL (203612 at) possible difference in mitochondrial content or mitochondrial activity in adipose tissue from lean subjects and overweight subjects. However, it can not be excluded that the increased mitochondrial activity actually reflects a difference in cell number in the adipose tissue biopsies, as increased adipose tissue mass can be due to increased adipocyte cell size as well as increased number of adipocytes.

The dietary intervention consisted of replacement of oleic acid and stearic acid in the placebo spread by shortchain polyunsaturated fatty acids (PUFA: C18:2 and
C18:3), conjugated linoleic acid (CLA: C18:2) and medium chain triglycerides (C12:0) in the intervention spread. Thus, the gene expression differences can be related to differences in intake of all these fatty acids and should be interpreted in that context.

Both CLA and PUFA (specifically n-3 PUFA) can exert anti-inflammatory effects [4, 24]. In the majority of overweight subjects, dietary intervention resulted in downregulation of expression of a number of inflammationrelated genes (in enriched category 'response to stress') 

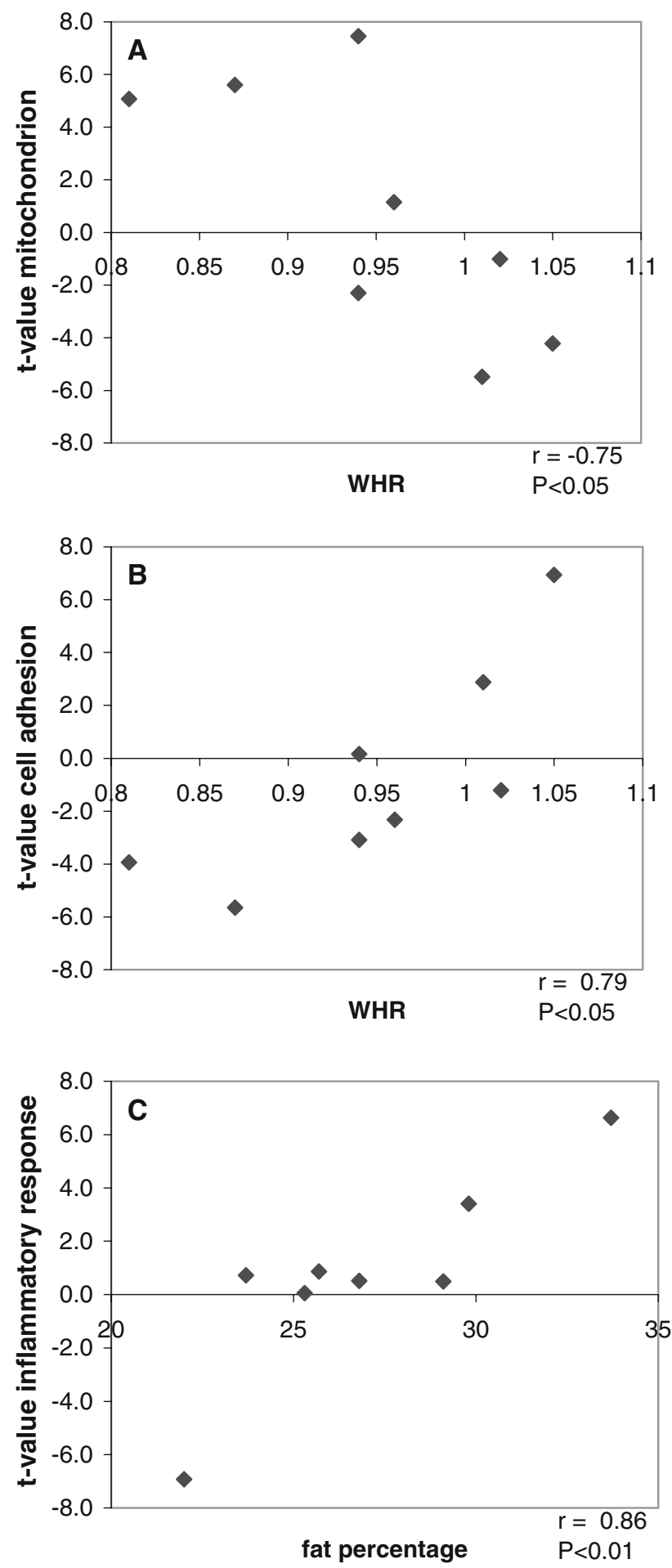

Fig. 4 Scatter plots of obesity phenotype parameters (waist-hip ratio $W H R$ or fat percentage) and scores ( $t$ value) for enrichment of functional processes (based on Gene Ontology) that were affected by dietary intervention. a Score for enrichment of genes encoding mitochondrial proteins for each overweight subject plotted against WHR; b Score for enrichment of cell adhesion genes plotted against WHR; c Score for enrichment of inflammatory response genes for each overweight subject plotted against fat percentage that were expressed at significantly higher level in adipose tissue from overweight subjects than in adipose tissue from lean subjects. This anti-inflammatory effect of the intervention was, e.g., seen for macrophage inflammatory protein 2 alpha (CXCL2), macrophage receptor MARCO (MARCO) and interleukin-1 receptor antagonist (IL1RN). In adipose tissue from lean subjects, the anti-inflammatory effect of the dietary intervention was restricted to downregulation of genes involved in complement activation pathway (three subunits of complement C1q) and genes involved in reactive oxygen species metabolism.

Additionally, in adipose tissue from lean subjects the dietary intervention resulted in a down-regulation of genes involved in energy metabolism-related processes (generation of precursor metabolites and energy, lipid metabolism, carbohydrate metabolism). Biological network analysis revealed that a number of the differentially expressed energy metabolism genes are targets of HNF4-alpha in liver and/or pancreas [25, 37]. Thus, possibly the expression of genes related to energy metabolism could have been regulated through transcription factor HNF4-alpha (Fig. 5). PUFA can bind to and repress activity of HNF4-alpha [11, 28, 37]. This implicates that, in addition to liver, HNF4alpha could be an important regulator of gene expression and energy metabolism in adipose tissue, at least in lean subjects.

In the majority of overweight subjects, dietary intervention affected expression of genes involved in lipid metabolism, resulting mostly in up-regulation of expression (Fig. 3). Expression of lipid metabolism genes was lower in adipose tissue from overweight subjects than in adipose tissue from lean subjects, therefore increased expression of lipid metabolism genes might be a beneficial effect of the intervention in overweight subjects. Moreover, increased energy expenditure as a result of up-regulation of lipid metabolism could be one of the mechanisms through which CLA decreases adipose tissue mass [4, 24, 37] and it could play a role in the proposed increase of energy expenditure by medium chain triglycerides [29]. Additional effects of the dietary intervention in the majority of overweight subjects included effects on genes involved in apoptosis and genes involved in cell adhesion and extracellular matrix. Effects on these processes are consistent with recently described effects of CLA and PUFA [9, 12, 37].

In overweight subjects the response to the dietary intervention was not as strong as in the lean subjects and moreover, the response among the overweight subjects was variable. The consistent response in a selection of overweight subjects was not related to treatment order. Possibly, adipose tissue in overweight subjects is not as flexible and responsive to dietary intervention as adipose 


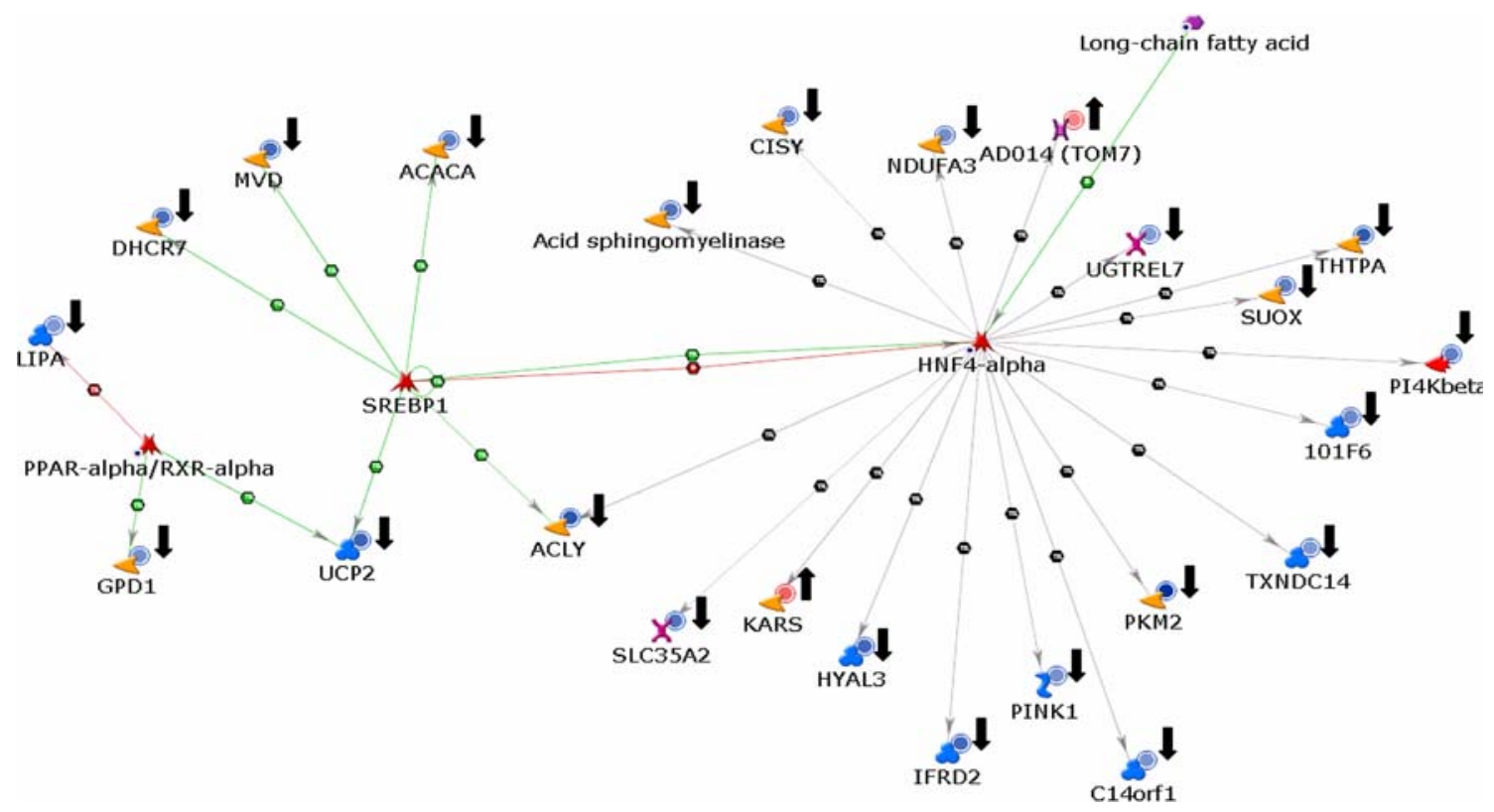

Fig. 5 Network of direct interactions between transcription factors HNF4alpha, SREBP1 and PPARA and genes involved in energy metabolism that were differentially expressed in lean subjects in response to intervention spread compared to control spread. Arrow pointing down indicates down-regulation of expression in response to intervention spread compared to control spread, arrow pointing up indicates up-regulation of expression in response to intervention spread compared to control spread. Lines between symbols indicate connections described in literature. This figure was made using $\operatorname{MetaCore}^{\mathrm{TM}}$ (v4.3) from GeneGo Inc. LIPA Lipase A, GPD1 glycerol3-phosphate dehydrogenase 1, UCP2 mitochondrial uncoupling protein 2, DHCR7 7-dehydrocholesterol reductase; $M V D$ diphosphomevalonate decarboxylase; $A C A C A$ acetyl-coA carboxylase $1 ; A C L Y$

tissue in lean subjects. Also, the intervention effect might have been diluted due to increased adipose tissue mass in overweight subjects compared to the lean subjects, therefore resulting in less pronounced expression changes. Lastly, genotype and polymorphisms can not be exluded as a factor in the variation in response.

The overweight subjects were selected based on BMI, an obesity marker that does not take fully into account adipose tissue distribution in the body. In contrast, WHR and waist circumference are more specific markers for body fat distribution and presence of central or upper-body obesity [15, 19]. Interestingly, the individual response of the overweight subjects to the intervention correlated to the obesity phenotype markers WHR and fat percentage. For example, expression of mitochondrial genes was up-regulated in response to dietary intervention in the overweight subject with the lowest WHR and down-regulated in response to intervention in the overweight subject with the highest WHR. Interestingly, these two subjects have a similar BMI. Overall, these results show that the subjects, classified as overweight on the basis of their BMI, are heterogeneous in their response to dietary intervention and that this response
ATP citrate lyase; CISY citrate synthase; NDUFA3 NADH dehydrogenase (ubiquinone) 1 alpha subcomplex, 3; AD014(TOM7) translocase of outer mitochondrial membrane 7 homolog (yeast); UGTREL7 UDP-glucuronic acid/UDP-N-acetylgalactosamine transporter; THTPA thiamine-triphosphatase; SUOX Sulfite oxidase, mitochondrial precursor; PI4Kbeta: Phosphatidylinositol 4-kinase beta; 101F6: Cytochrome b561 domain-containing protein 2; TXNDC14: thioredoxin domain containing 14; PKM2 Pyruvate kinase 2/3; C14orf1: Probable ergosterol biosynthetic protein 28; PINK1 Serine/threonine-protein kinase PINK1, mitochondrial precursor; IFRD2 Interferon-related developmental regulator 2; HYAL3 Hyaluronidase-3 precursor; KARS Lysyl-tRNA synthetase; SLC35A2 UDPgalactose translocator

is associated with obesity phenotype markers WHR and fat percentage, suggesting a link between body fat distribution and response to nutritional intervention.

The fat biopsies analyzed were subcutaneous biopsies, taken from the abdomen. Abdominal fat, mostly visceral abdominal fat, is often linked to complications and disease progression [36]. However, some studies report a role for subcutaneous abdominal tissue in obesity related diseases [10]. The finding that subcutaneous adipose tissue responds to nutritional intervention is encouraging for further research, as this is the only easily accessible source of adipose tissue from humans. Thus, whole genome expression profiling in adipose tissue may help to identify relevant mechanisms and targets of nutritional intervention in obesity prevention or treatment.

In summary, response to the dietary intervention was different in lean and overweight subjects. Increased short-chain polyunsaturated fatty acids (PUFA: C18:2 and C18:3), conjugated linoleic acid (CLA: C18:2) and medium chain triglycerides (C12:0) consumption resulted in lower expression of genes related to energy metabolism in lean subjects, whereas in the majority of 
overweight subjects expression of inflammatory genes was down-regulated and expression of lipid metabolism genes was up-regulated. Individual responses in overweight subjects were variable and these correlated better to obesity phenotype markers WHR and fat percentage than BMI.

Acknowledgments The authors express their gratitude to the volunteers that participated in the study, to the people from the Metabolic Research Unit and laboratories who assisted in the organization of the study and to Michèle van den Wijngaard for RNA isolation. The authors are member of the European Nutrigenomics Organization (www.nugo.org). This study was financially supported by Dutch government through grant "Healthy nutrition" (grant number 04003).

Conflict of interest statement None of the authors has any conflict of interest.

Open Access This article is distributed under the terms of the Creative Commons Attribution Noncommercial License which permits any noncommercial use, distribution, and reproduction in any medium, provided the original author(s) and source are credited.

\section{References}

1. Berg AH, Scherer PE (2005) Adipose tissue, inflammation, and cardiovascular disease. Circ Res 96:939-949

2. Boorsma A, Foat BC, Vis D, Klis F, Bussemaker HJ (2005) Tprofiler: Scoring the activity of predefined groups of genes using gene expression data. Nucleic Acids Res 33:W592-W595

3. Brown JM, McIntosh MK (2003) Conjugated linoleic acid in humans: regulation of adiposity and insulin sensitivity. $\mathrm{J}$ Nutr 133:3041-3046

4. Browning LM (2003) n-3 polyunsaturated fatty acids, inflammation and obesity-related disease. Proc Nutr Soc 62:447-453

5. Calder PC (2006) n-3 Polyunsaturated fatty acids, inflammation, and inflammatory diseases. Am J Clin Nutr 83

6. Dahlman I, Linder K, Nordstrom EA, Andersson I, Liden J et al (2005) Changes in adipose tissue gene expression with energyrestricted diets in obese women. Am J Clin Nutr 81:1275-1285

7. Doniger SW, Salomonis N, Dahlquist KD, Vranizan K, Lawlor SC, Conklin BR (2003) MAPPFinder: using Gene Ontology and GenMAPP to create a global gene-expression profile from microarray data. Genome Biol 4:R7

8. Eisen MB, Spellman PT, Brown PO, Botstein D (1998) Cluster analysis and display of genome-wide expression patterns. Proc Natl Acad Sci USA 95:14863-14868

9. Fischer-Posovszky P, Kukulus V, Zulet MA, Debatin KM, Wabitsch M (2007) Conjugated linoleic acids promote human fat cell apoptosis. Horm Metab Res 39:186-191

10. Goodpaster BH, Thaete FL, Simoneau JA, Kelley DE (1997) Subcutaneous abdominal fat and thigh muscle composition predict insulin sensitivity independently of visceral fat. Diabetes 46:1579-1585

11. Hertz R, Magenheim J, Berman I, Bar-Tana J (1998) Fatty acyl$\mathrm{CoA}$ thioesters are ligands of hepatic nuclear factor-4a. Nature 392:512-516

12. Huber J, Löffler M, Bilban M, Reimers M, Kadl A et al (2007) Prevention of high-fat diet-induced adipose tissue remodeling in obese diabetic mice by $n-3$ polyunsaturated fatty acids. Int $J$ Obes 31:1004-1013
13. Kamphuis MMJW, Lejeune MPGM, Saris WHM, WesterterpPlantenga MS (2003) Effect of conjugated linoleic acid supplementation after weight loss on appetite and food intake in overweight subjects. Eur J Clin Nutr 57:1268-1274

14. Kern PA, Di Gregorio GB, Lu T, Rassouli N, Ranganathan G (2003) Adiponectin expression from human adipose tissue: relation to obesity, insulin resistance, and tumor necrosis factor-? expression. Diabetes 52:1779-1785

15. Kok P, Seidell JC, Meinders AE (2004) The value and limitations of the Body Mass Index (BMI) in the assessment of the health risks of overweight and obesity. Ned Tijdschr Geneeskd 148: 2379-2382

16. Kolaczynski JW, Morales LM, Moore J, Considine RV, Pietrzkowski $\mathrm{Z}$ et al (1994) A new technique for biopsy of human abdominal fat under local anaesthesia with Lidocaine. Int J Obes 18:161-166

17. Kovacs EMR, Mela DJ (2006) Metabolically active functional food ingredients for weight control. Obes Rev 7:59-78

18. Lawton CL, Delargy HJ, Brockman J, Smith FC, Blundell JE (2000) The degree of saturation of fatty acids influences postingestive satiety. Br J Nutr 83:473-482

19. Lev-Ran A (2001) Human obesity: an evolutionary approach to understanding our bulging waistline. Diabetes Metab Res Rev 17:347-362

20. Lyons PA, Koukoulaki M, Hatton A, Doggett K, Woffendin HB et al (2007) Microarray analysis of human leucocyte subsets: the advantages of positive selection and rapid purification. BMC Genomics 8:64

21. Madsen L, Petersen RK, Kristiansen K (2005) Regulation of adipocyte differentiation and function by polyunsaturated fatty acids. Biochimica et Biophysica Acta Mol Basis Dis 1740:266286

22. Mangravite LM, Dawson K, Davis RR, Gregg JP, Krauss RM (2007) Fatty acid desaturase regulation in adipose tissue by dietary composition is independent of weight loss and is correlated with the plasma triacylglycerol response. Am J Clin Nutr 86:759767

23. Mapfei M, Halaas J, Ravussin E, Pratley RE, Lee GH et al (1995) Leptin levels in human and rodent: Measurement of plasma leptin and ob RNA in obese and weight-reduced subjects. Nat Med $1: 1155-1161$

24. Moloney F, Toomey S, Noone E, Nugent A, Allan B et al (2007) Antidiabetic effects of cis-9, trans-11-conjugated linoleic acid may be mediated via anti-inflammatory effects in white adipose tissue. Diabetes 56:574-582

25. Odom DT, Zizlsperger H, Gordon DB, Bell GW, Rinaldi NJ et al (2004) Control of Pancreas and Liver Gene Expression by HNF Transcription Factors. Science 303:1378-1381

26. Pietilainen KH, Naukkarinen J, Rissanen A, Saharinen J, Ellonen $P$ et al (2008) Global transcript profiles of fat in monozygotic twins discordant for BMI: pathways behind acquired obesity. PLoS Med 5:e51

27. Ronti T, Lupattelli G, Mannarino E (2006) The endocrine function of adipose tissue: An update. Clin Endocrinol 64:355-365

28. Sampath H, Ntambi JM (2005) Polyunsaturated fatty acid regulation of genes of lipid metabolism. Annu Rev Nutr 25:317-340

29. St-Onge MP, Jones PJH (2002) Physiological effects of mediumchain triglycerides: Potential agents in the prevention of obesity. J Nutr 132:329-332

30. Su AI, Wiltshire T, Batalov S, Lapp H, Ching KA et al (2004) A gene atlas of the mouse and human protein-encoding transcriptomes. Proc Natl Acad Sci USA 101:6062-6067

31. Subramanian A, Tamayo P, Mootha VK, Mukherjee S, Ebert BL et al (2005) Gene set enrichment analysis: A knowledge-based approach for interpreting genome-wide expression profiles. Proc Natl Acad Sci USA 102:15545-15550 
32. van Erk MJ, Blom WA, van Ommen B, Hendriks HF (2006) High-protein and high-carbohydrate breakfasts differentially change the transcriptome of human blood cells. Am J Clin Nutr 84:1233-1241

33. Van Strien T, Frijters JER, Bergers GPA, Defares PB (1986) The Dutch eating behavior questionnaire (DEBQ) for assessment of restrained, emotional, and external eating behavior. Int $\mathrm{J}$ Eat Disord 5:295-315

34. Van Strien T, Frijters JER, Bergers GPA, Defares PB (1986) Nederlandse vragenlijst voor eetgedrag Lisse, Zwets and Zeitlinger b.v

35. Viguerie N, Vidal H, Arner P, Holst C, Verdich C et al (2005) Adipose tissue gene expression in obese subjects during low-fat and high-fat hypocaloric diets. Diabetologia 48:123-131
36. Wajchenberg BL (2000) Subcutaneous and visceral adipose tissue: Their relation to the metabolic syndrome. Endocr Rev 21:697-738

37. Wang YW, Jones PJH (2004) Conjugated linoleic acid and obesity control: Efficacy and mechanisms. Int J Obes 28:941-955

38. Weisberg SP, McCann D, Desai M, Rosenbaum M, Leibel RL, Ferrante J (2003) Obesity is associated with macrophage accumulation in adipose tissue. J Clin Invest 112:1796-1808

39. Wellen KE, Hotamisligil GS (2003) Obesity-induced inflammatory changes in adipose tissue. J Clin Invest 112:1785-1788

40. Whigham LD, Watras AC, Schoeller DA (2007) Efficacy of conjugated linoleic acid for reducing fat mass: A meta-analysis in humans. Am J Clin Nutr 85:1203-1211 